\title{
La encefalopatía espongiforme: ¿un trastorno conversivo?
}

Spongiform encephalopathy: a conversive disorder?

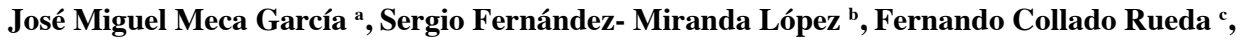 \\ Fernando Vázquez Gutiérrez ${ }^{\text {d. }}$
}

${ }^{a}$ MIR Psiquiatría. UGC SM Almería, España. ${ }^{b}$ FEA Psiquiatría UGC SM Almería, España. ${ }^{c}$ MIR Psiquiatría. UGC SM Almería, España. ${ }^{d}$ FEA Neurología UGC Neurología Almería, España.

Correspondencia: José Miguel Meca García (mecaarrebola@gmail.com)

Recibido: 24/11/2013; aceptado: 26/01/2014

RESUMEN: Las prionpatías, constituyen un grupo de enfermedades neurodegenerativas con presentación familiar, esporádica o adquirida, con clínica heterogenea y resultado fatal. Los síntomas psiquiátricos pueden estar presentes al inicio y/o durante la evolución del cuadro, con la presencia o no de focalidad neurológica. Esta condición conlleva a que estos pacientes sean derivados a unidades de Psiquiatría tanto a nivel hospitalario como ambulatorio, para estudio de trastornos psiquiátricos. El caso clínico que nos ocupa el paciente fue atendido en varias ocasiones en las Urgencias Hospitalarias, evaluado por neurólogos y psiquiatras con diversos diagnósticos psiquiátricos. Sin embargo, la encefalopatía espongiforme sólo fue diagnosticada en el último mes de enfermedad.

PALABRAS CLAVE: trastorno disociativo, trastorno conversivo, encefalopatía espongiforme.
ABSTRACT: Prionpathies represent a group of neurodegenerative diseases of familiar, sporadic or acquired origin, heterogeneous symptoms and fatal outcome. The psychiatric symptoms can be present at the beginning and/or during the clinical evolution, with the presence or not of neurological disorders. Patients with these sympthoms are referred to psychiatric units, both at hospital level and as outpatients, to study a psychiatric disorder. In the described clinical case, the patient was admitted several times at emergency units, rated by neurologists and psychiatrists leading to different psychiatric disorders diagnoses. However spongiform encephalopathy was diagnosed only during the last month of the disease.

KEY WORDS: dissociative disorder, conversive disorder, spongiform encephalopathy.

\section{Introducción.}

Las enfermedades por priones son un grupo de enfermedades neurodegenerativas que se presentan de manera familiar, adquirida y esporádica. Son trastornos de la conformación de las proteínas, que se manifiestan como enfermedades neurodegenerativas en los humanos. La acumulación de $\operatorname{PrP}^{\mathrm{sc}}$ (isoforma alterada de la $\mathrm{PrP}^{\mathrm{c}}$ ) produce afectación de la sustancia gris con muerte neuronal, proliferación astrocitaria y cambios espongiformes. El denominado amiloide priónico se deposita en forma de placas en el encéfalo, y se origina como consecuencia de una proteólisis incompleta de la $\mathrm{PrP}^{\mathrm{sc}}$. La proteína priónica (PrP) está codificada por el gen de la proteína priónica (PRNP) del brazo corto del cromosoma 20 cuyo polimorfismo marcará el tipo de enfermedad por priones que padece el paciente.(1) 
Según el Registro Nacional de Encefalopatías Espongiformes Transmisibles Humanas, a 31 de diciembre de 2010 en España se notificaron 1.292 casos descartándose 222. La más frecuente ha sido la enfermedad de Creutzfeldt-Jacob (ECJ), con 533 casos confirmados, 380 casos probables y 47 posibles. De la variante de ECJ(vECJ) cinco casos, 48 casos de Insomnio Familiar Letal(IFL) y dos del Síndrome de Gerstmann-Sträussler-Scheinker(SGSS).(2)

El diagnóstico de la ECJ se basa en los hallazgos clínicos, en combinación con los resultados de un determinado tipo de pruebas complementarias como el electroencefalograma(EEG), examen del LCR y las pruebas de neuroimagen, concretamente en Resonancia Magnética Nuclear(3-4).

Clínicamente es un cuadro muy heterogéneo, pero con resultados fatales, esta variabilidad clínica tal vez tenga su base en la distribución de las lesiones microscópicas espongiformes en distintas aéreas cerebrales y por el polimorfismo cromosomico $(5,8)$. La presencia de clínica psiquiátrica o la dificultad de considerar el cuadro como neurológico en los inicios de la sintomatología tanto clínicamente como por la normalidad de pruebas complementarias $(6-7,10)$, puede llevar a estos pacientes a seguimiento en unidades de Psiquiatría tanto a nivel hospitalario como comunitario. La sintomatología psiquiátrica es variada, habiéndose descrito cambios en la personalidad, síntomas en la esfera psicótica, depresión, ansiedad, insomnio, conversión, disociación (6-10).

\section{Presentación del caso:}

Paciente de 60 años sin antecedentes psiquiátricos de interés, antecedentes de transplante de cornea de ojo izquierdo e intervenido de hernia inguinal. El paciente contacta con Urgencias para evaluar la presencia de una hipoacusia bilateral de dos semanas de evolución, refiriendo una sensación de taponamiento semejante a la sensación de subir a una gran altura, acompañado según refería, de dificultad para la comprensión de la lectura y del lenguaje, todo ello con cierta fluctuación, y que no se evidenció en la consulta. En los últimos meses el paciente había comenzado con problemas de sueño en relación a preocupaciones laborales y problemas de pareja. El paciente fue remitido a Otorrinolaringología para estudio de hipoacusia con resultado de normalidad en la exploración y en la audiometría. A la exploración no presentó focalidad neurológica evidenciable, solicitando TAC sin contraste sin evidenciar hallazgos anormales. Tras consultar con Neurología descartan cuadro de etiología neurológica, estimándose la posibilidad de cuadro de características conversivas en relación a los eventos vitales estresantes. El paciente es derivado a Atención Primaria y a seguimiento por Psiquiatría si fuera necesario. 
NOTAS CLÍNICAS

A las dos semanas de la primera admisión por Urgencias acude para nueva valoración urgente ante la persistencia de la sintomatología previa e incremento dificultades para sueño. Es citado para estudio ambulatorio por Neurología y Psiquiatría.

El paciente inicia seguimiento en Psiquiatría en consultas con orientación diagnostica de posible cuadro disociativo e inicio de tratamiento con Fluoxetina $20 \mathrm{mg}$ y Lormetazepám $1 \mathrm{mg}$. Es seguido en consulta de Neurología donde se solicita estudio de RMN sin hallazgos de interés y con orientación diagnóstica a cuadro conversivo.

Al mes de evolución el paciente vuelve a contactar con servicio de Urgencias por empeoramiento progresivo del cuadro, con alteración sobreañadida de la marcha, habiendo mejorado el sueño con la benzodiazepinas. Nuevamente valorado por Neurología, destaca una rigidez paratónica, con reflejos miotáticos normales y sin piramidalismo, confirmando la idea de cuadro no neurológico. En esa asistencia es valorado por Psiquiatría destacando en la exploración un mutismo, afasia global, que el paciente intentaba explicar con gestos y una torpeza en la deambulación, sin impresionar de ataxia clara, con sueño y apetito conservados, sin poder evaluar otras esferas. Se propone a la familia que en caso de que el cuadro evolucionara y no pudieran proporcionarle los cuidados adecuados se propondría ingreso en la Unidad de Hospitalización de Psiquiatría para estudio y tratamiento. Se pauta Olanzapina 5mg.

A la semana de la última asistencia, el paciente es ingresado en Psiquiatría con juicio clínico de Trastorno Conversivo.

En el ingreso el paciente permanece mutista, negativista, no colaborador, inhibido en sus necesidades básicas, con alteración en la marcha, haciéndose imposible en momentos, aunque de manera fluctuante y espontánea el paciente anda y come. La evolución tiende al mutismo, mantenimiento de posturas antigravitatorios en ocasiones y en otras sin apreciar una rigidez evidente. Las observaciones del personal de enfermería alimentan la confusión del cuadro y el patrón evolutivo poco lógico. En el transcurso de una semana la evolución se agrava con una tendencia a un sopor precomatoso con la presencia de una rigidez generalizada de características paratónicas, ausencia de reflejo visual de amenaza, mioclonías braquiales, incontinencia urinaria y fecal. Ingresando en planta de Neurología.

El resultado de la RMN que se repite aproximadamente al mes de la anterior, muestra un patrón compatible con encefalopatía espongiforme, con lesiones bilaterales y simétricas en núcleos caudados y porción anterior del putamen, con alteración en la señal de la cortical de lóbulos temporales y parietales. El EEG realizado en vigilia, se aprecia una actividad de fondo alterada, con enlentecimiento de actividad de fondo, compuesta por frecuencias theta generalizadas, sobre esta frecuencia de fondo se observan presencia de FIRDA (actividad delta rítmica inter- 
mitente), todo ello concordante con una afectación cerebral generalizada de grado moderado, sin apreciarse anomalías paroxísticas epileptiformes, ni eventos críticos. La evolución del paciente se complica con cuadro de hepatitis aguda y neumonía posiblemente aspirativa, precoma y finalmente coma. Complementándose el estudio y tratamiento empírico para descartar Wilson y encefalitis inmunomediadas. Serologías de VIH, VHB y TP negativas, marcadores tumorales negativos, metales pesados y tóxicos en orina negativas, B12 normal y perfil tiroideo negativo, El estudio de punción lumbar descarta TBC y virus neurotropos, TAC toracoabdominal sin alteraciones, y secuenciación del gen de la proteína priónica con resultado de genotipo homocigoto para la posición codónica $129 \operatorname{Met} 129 \operatorname{Met}(\mathrm{p} .129 \mathrm{M} / \mathrm{M})$, indicador de factor de riesgo para ECJ esporádico.

El deterioro motor y cognitivo fue rápidamente progresivo desde su primer contacto con Urgencias, en cuestión de menos de 5 meses hasta el fallecimiento del paciente.

\section{Discusión.}

La clínica psiquiátrica presente en la encefalopatía espongiforme, en ocasiones no acompañada de focalidad neurológica, incluso con presencia de pruebas de neuroimagen y neurofisiológicas normales en los inicios (6-7, 9), o incongruentes con la sintomatología presentada (4) conllevando cuadros confusos que tras descartar origen neurológico o de otra índole, termina cayendo en manos de los Psiquiatras, con diagnósticos de trastornos de la personalidad, somatomorfos, conversivos, disociativos, psicóticos de la esfera ansiosa o afectiva (6-10). El psiquiatra como receptor final de estos pacientes deberá estar avezado en hacer diagnóstico diferenciales adecuados para evitar una línea de tratamiento inadecuado, partiendo de una base de error diagnóstica. El Psiquiatra deberá cuestionar de manera razonable y basándose en sus conocimientos sobre la enfermedad mental y de otras aéreas de la medicina, los diagnósticos psiquiátricos procedentes de otros especialistas, basados en el descarte por la inexistencia de datos objetivables a través de pruebas complementarias, pruebas con muchas limitaciones a la vista de lo tratado en este artículo $(6-7,9)$. 


\section{Conclusiones.}

Por suerte las prionpatías son un grupo de enfermedades muy poco frecuentes, pero que en la práctica médica deben ser tenidas en cuenta. La Psiquiatría debe estar preparada para poder filtrar este tipo de enfermedades. La sintomatología psiquiátrica y el diagnóstico de trastorno psiquiátrico en el inicio de la enfermedad es posible.

La variabilidad de síntomas que pueden surgir, está posiblemente en relación a las lesiones histopatologías extendidas en distintas aéreas cerebrales, sin detrimento de la posible elaboración por parte del paciente de su situación vital, que tal vez en función de las lesiones invisibles pudieran exhibir o liberar las defensas más inmaduras, que pudieran confundir más aún el diagnóstico.

\section{BIBLIOGRAFÍA.}

(1) Sadock BJ, Sadock VA. Kaplan\&Sadock. Sinopsis de Psiquiatría. 10ªed. Baltimore, MD. Wolters Kluwer, Lippincott Willians \& Wilkins.2009.

(2) Centro Nacional de Epidemiología. Instituto Carlos III. Resultados de la vigilancia epidemiológica de las enfermedades transmisibles. Informe anual. Madrid. 2012:88-90.

(3) Arranz Martínez E, Trillo Sánchez Redondo G, Ruiz García A, Ares Blanco S. Prionpatias: las encefalopatías por priones. Semergen. 2010; 36(8):443-448.

(4) Toribio Díaz ME, García Di Ruggiero E, Pérez Parra F. Dos nuevos casos de Enfermedad de Creutzfeldt Jakob en Madrid, España. Rev Neurol.2011; 53(5): 281-286.

(5) Solvason HB, Harris B, Zeifert P, Flores BH, Hayward C. Psychological versus Biological Clinical Interpretation: A Patient With Prion Disease. Am J Psychiatry. 2002;159:528-537.

(6) Estevens EM, Lament R. Psychiatric Presentation of Jakob-Creutzfeldt Disease. J Clin Psychiatry.1979; 40:445-446.

(7) Keshavan et al. Psychiatric Presentation of Creutzfeldt-Jakob Disease. A Case Report. British Journal of Psychiatry.1987;151:260-263.

(8) Power B, Trivedi D, Samuel M. What psychiatrists should know about sporadic Creutzfeldt-Jakob disease. Australasian Psychiatry. 2012;20(1): 61-66.

(9) Jardri R, Dipaola C, Lajugie C, Thomas P, Goeb JL. Depressive disorder with psychotic symptoms as psychiatric presentation of sporadic Creutzfeldt-Jakob disease: a case report. General Hospital Psychiatry. 2006; 28(5): 452-454.

(10) Allrogen H, Dennis G, Abbott RJ, Pye IF. New variant Creutzfeldt-Jakob disease: three case reports from Leicestershire. J Neurol Neurosurg Psychiatry. 2000; 68: 375-378. 